\title{
The Genus Caulerpa from Central Visayas, Philippines
}

\section{ERNANI G. MEÑEZ \\ and}

\section{HILCONIDA P. GALUMPONG}




\section{SERIES PUBLICATIONS OF THE SMITHSONIAN INSTITUTION}

Emphasis upon publication as a means of "diffusing knowledge" was expressed by the first Secretary of the Smithsonian. In his formal plan for the Institution, Joseph Henry outlined a program that included the following statement: "It is proposed to publish a series of reports, giving an account of the new discoveries in science, and of the changes made from year to year in all branches of knowledge." This theme of basic research has been adhered to through the years by thousands of titles issued in series publications under the Smithsonian imprint, commencing with Smithsonian Contributions to Knowledge in 1848 and continuing with the following active series:

\section{Smithsonian Contributions to Anthropology \\ Smithsonian Contributions to Astrophysics \\ Smithsonian Contributions to Botany \\ Smithsonian Contributions to the Earth Sciences \\ Smithsonian Contributions to the Marine Sciences \\ Smithsonian Contributions to Paleobiology \\ Smithsonian Contributions to Zoology \\ Smithsonian Studies in Air and Space \\ Smithsonian Studies in History and Technology}

In these series, the Institution publishes small papers and full-scale monographs that report the research and collections of its various museums and bureaux or of professional colleagues in the world of science and scholarship. The publications are distributed by mailing lists to libraries, universities, and similar institutions throughout the world.

Papers or monographs $\$$ ubmitted for series publication are received by the Smithsonian Institution Press, subject to its own review for format and style, only through departments of the various Smithsonian museums or bureaux, where the manuscripts are given substantive review. Press requirements for manuscript and art preparation are outlined on the inside back cover.

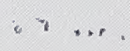

S. Dillon Ripley

Secretary

Smithsonian Institution 


\title{
The Genus Caulerpa from Central Visayas, Philippines
}

\author{
Ernani G. Meñez \\ and Hilconida P. Calumpong \\ ISSUED \\ OCT 51982
}

SMITHSONIAN PUBLICATIONS

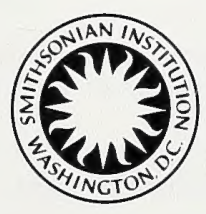

SMITHSONIAN INSTITUTION PRESS

City of Washington

1982 


\section{A B S T R A C T}

Meñez, Ernani G., and Hilconida P. Calumpong. The Genus Caulerpa from Central Visayas, Philippines. Smithsonian Contributions to the Marine Sciences, number 17, 21 pages, 2 figures, 3 plates, 1982.-This taxonomic study of Caulerpa shows 20 taxa occurring in Central Visayas, Philipines, including Caulerpa reyesii, new species. Of these, three are newly reported from the Philippines and seven taxa are new records from Central Visayas.

OfFicial pUblication DATE is handstamped in a limited number of initial copies and is recorded in the Institution's annual report, Smithsonian Year. Series cover Design: Seascape along the Atlantic coast of eastern North America.

Library of Congress Cataloging in Publication Data Menez, Ernani G.

The genus Caulerpa from central Visayas, Philippines

(Smithsonian contributions to the marine sciences; no. 17)

Bibliography: p.

1. Caulerpa-Classification. 2. Botany-Philippines-Visayan Islands-Classification. I. Calumpong, Hilconida P. II. Title III. Series. QK569.C37M46 $1982 \quad 589.4^{\prime} 7 \quad 82-5976 \quad$ AACR2 


\section{Contents}

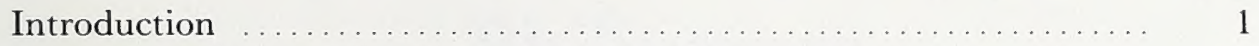

Acknowledgments ............................... 3

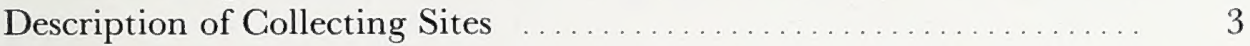

Class Chlorophyceae ............................... 4

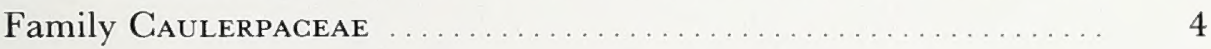

Genus Caulerpa .................................. 4

Key to Species of Caulerpa from Central Visayas ........... 4

Caulerpa arenicola Taylor ........................... 5

Caulerpa brachypus Harvey ........................... 5

Caulerpa crassifolia (C. Agardh) J. Agardh ............... 6

Caulerpa cupressoides (West in Vahl) C. Agardh ............. 6

Caulerpa fastigiata Montagne ......................... 6

Caulerpa lentillifera J. Agardh ....................... 7

Caulerpa microphysa (Weber-van Bosse) Feldmann ........... 7

Caulerpa racemosa (Forsskål) J. Agardh .................. 7

Caulerpa racemosa var. clavifera (Turner) Weber-van Bosse ...... 7

Caulerpa racemosa var. macrophysa (Kützing) Taylor ........... 8

Caulerpa racemosa var. occidentalis (J. Agardh) Børgesen ........ 8

Caulerpa racemosa var. peltata (Lamouroux) Eubank ........... 8

Caulerpa racemosa var. uvifera (Turner) J. Agardh ........... 9

Caulerpa serrulata (Forsskål) J. Agardh .................. 9

Caulerpa serrulata var. boryana f. occidentalis (Weber-van Bosse)

Yamada and Tanaka ............................. 9

Caulerpa sertularioides (Gmelin) Howe .................. 9

Caulerpa urvilliana Montagne ........................ 10

Caulerpa webbiana Montagne ......................... 10

Caulerpa verticillata J. Agardh ....................... 10

Caulerpa reyesii, new species ........................ 10

Literature Cited ..................................... 13

Plates ............................................ 15 



\section{The Genus Caulerpa from Central Visayas, Philippines}

\section{Ernani G. Meñez and Hilconida P. Calumpong}

\section{Introduction}

During the summers of 1978 and 1979, Smithsonian Institution conducted biological collecting expeditions in Central Visayas, Philippines, in order to collect marine plant and animal organisms for purposes of systematic, ecological, and distributional studies. Included in the plant collection was a large number of Caulerpa species, which forms the basis of the present study, taken from different parts of this region.

Fifty-three taxa of Caulerpa have been previously reported from the Philippines. Of these, 14 occurred in Central Visayas. These were Caulerpa brachypus, C. cupressoides, C. fastigiata, C. lentillifera, C. peltata, C. racemosa, C. racemosa var. laetevirens, $C$. racemosa var. macrophysa, $C$. serrulata, $C$. serrulata var. typica f. lata, C. sertularioides, C. taxifolia, $C$. urvilliana, and $C$. verticillata. A few of these taxa were not found in our collections.

The principal collecting areas are in Central Visayas, in the middle of the Philippine Archipelago (Figure 1). The area consists of the islands of Negros, Cebu, Bohol, Siquijor, and their satellite islets. Except for the southwestern portion

Ernani G. Meñez, Smithsonian Oceanographic Sorting Center, Smithsonian Institution, Washington, D.C. 20560. Hilconida P. Calumpong, Department of Biology, Silliman University, Dumaguete City 6501 , Philippines. of Negros Island, which is exposed to a major body of water, the Sulu Sea, the rest of Central Visayas is sheltered. Because it is practically closed off by other surrounding islands, the greater portion of this area is uniquely protected from intense wave actions and currents, thus, Central Visayas may represent a major local phytogeographic boundary in the Philippines. In an unpublished thesis by Calumpong (1981), she recorded 216 taxa of benthic marine algae from Central Visayas. Of these, 50 taxa are newly reported from the Philippines.

The first detailed study on Philippine Caulerpa was by Gilbert (1942), who reported 10 taxa but only Caulerpa serrulata var. typica f. lata was recorded as occurring in Mactan and Cebu Islands, Central Visayas. Later, Taylor (1966) listed two varieties of Caulerpa racemosa from Cebu Island. However, the bulk of the work on algae from Central Visayas was done by Reyes $(1972,1976)$, who collected from the islands of Negros and Siquijor and described 11 taxa of Caulerpa from this region.

The purpose of this study is to identify the Caulerpa species occurring in Central Visayas, Philippines, and to provide a sound reference and a simple, workable key for the identification of these species. Twenty taxa are described, includ- 


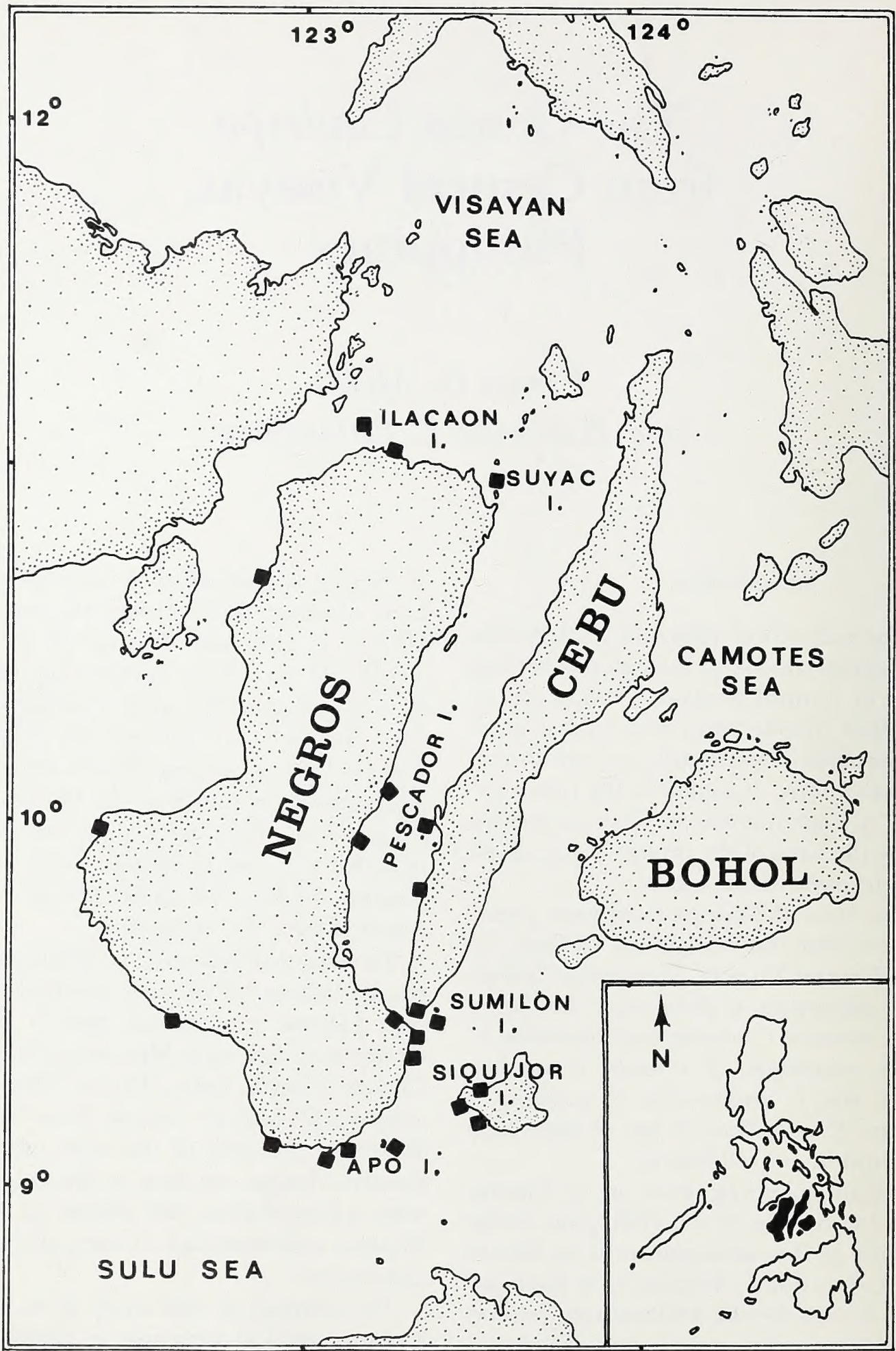

Figure 1.-Map of Central Visayas, Philippines; inset is general map of Philippines showing location of Central Visayas (black squares = collecting sites). 
ing Caulerpa reyesii, new species. Of these, three are newly reported from the Philippines and seven taxa are new records from Central Visayas. The taxa new to the Philippines are designated with one asterisk (*) and those from Central Visayas are marked with two asterisks $(* *)$ in the systematic section. Basionyms and synonyms are included in the list. References pertinent to the study of Caulerpa, general collection data, and distribution records in the Philippines are included. Collector is designated as EM for E.G. Meñez. The numbers preceding the collector correspond to the year of collection. The collecting site number appears after the collector and this is followed by specimen numbers in parentheses. Specimens are being deposited in the U.S. National Herbarium, Smithsonian Institution, the Silliman University Herbarium in Dumaguete City, Philippines, and the Philippine National Herbarium in Manila, Philippines.

Acknowledgments.-Ernani G. Meñez acknowledges the financial support of the Smithsonian Institution Fluid Research Fund in the Philippines for travel and research. Hilconida P. Calumpong is grateful for the one-year study leave and travel assistance provided by the Silliman University Faculty and Staff Development Program through Dr. Angel C. Alcala. Ms. Calumpong's research in the USA was made possible through a fellowship award from the Smithsonian Institution Educational Outreach Program.

We are indebted to Dr. William J. Gilbert, Department of Biology, Albion College, to Dr. Robert T. Wilce, Department of Botany, University of Massachusetts, and to Dr. Roy T. Tsuda, The Marine Laboratory, University of Guam, for their critical review of this paper.

We appreciate the help of Dr. Harold E. Robinson, Department of Botany, Smithsonian Institution, in the Latin description of Caulerpa reyesii, new species. We also wish to thank Dr. Paul C. Silva, Curator, University of California (Berkeley) Herbarium for the use of the Index Nominum Algarum card file and his assistance with problems of literature citations.
The loan of herbarium material from the U.S. National Herbarium, Smithsonian Institution and Silliman University Herbarium, Philippines, through the kindness of Dr. James Norris and Prof. Alfredo Reyes, respectively, is gratefully acknowledged.

The bulk of the material used for this study was provided by the Smithsonian Oceanographic Sorting Center. The material was collected during the 1978 and 1979 Smithsonian Institution marine biological expeditions to Central Visayas, Philippines. The expeditions were organized by the Smithsonian Oceanographic Sorting Center and supported by the Smithsonian Scholarly Studies Program and Dreyfuss Foundation.

\section{Description of Collecting Sites}

78 EM-1:Liloan Point, southern Cebu $\left(9^{\circ} 25^{\prime} 15^{\prime \prime} \mathrm{N}, 123^{\circ}\right.$. $\left.18^{\prime} 10^{\prime \prime} \mathrm{E}\right)$. Algae collected from seagrass bed and coral reef, 0.5-3.0 m, 8 May 1978 .

78 EM-3: Paliton, San Juan, Siquijor Island $\left(9^{\circ} 10^{\prime} 25^{\prime \prime} \mathrm{N}\right.$, $\left.123^{\circ} 28^{\prime} 15^{\prime \prime} \mathrm{E}\right)$. Algae collected from an extensive seagrass bed and from a back reef with isolated clumps of corals, $0.2-6.5 \mathrm{~m}, 9$ May 1978.

78 EM-5: Solong-on, Siquijor town, Siquijor Island $\left(9^{\circ} 13^{\prime} 00^{\prime \prime} \mathrm{N}, 123^{\circ} 27^{\prime} 30^{\prime \prime} \mathrm{E}\right)$. Algae collected from seagrass bed, 0.5-3.0 m, 10 May 1978.

78 EM-7: Tambobo, Siaton, Negros Oriental $\left(9^{\circ} 03^{\prime} 45^{\prime \prime} \mathrm{N}\right.$, $\left.123^{\circ} 07^{\prime} 33^{\prime \prime} \mathrm{E}\right)$. Algae collected from isolated limestone rocks inside the bay, 1.5-3.0 m, 12 May 1978.

78 EM-9: Bantayan Beach, Dumaguete City, Negros Oriental $\left(9^{\circ} 22^{\prime} 30^{\prime \prime} \mathrm{N}, 123^{\circ} 15^{\prime} 45^{\prime \prime} \mathrm{E}\right)$. Algae collected from isolated rocks and seagrasses, 1.0-2.0 m, 15 May 1978.

78 EM-10: Lag-it, Okiot, Bais, Negros Oriental $\left(9^{\circ} 35^{\prime} 46^{\prime \prime} \mathrm{N}\right.$, $\left.123^{\circ} 09^{\prime} 24^{\prime \prime} \mathrm{E}\right)$. Algae collected from seagrass beds and corals, 1.0-2.0 m, 17 May 1978.

78 EM-22: Linaon, about $8 \mathrm{~km}$ south of Sojoton Point, Negros Occidental ( $9^{\circ} 57^{\prime} 06^{\prime \prime}$ N, $\left.122^{\circ} 26^{\prime} 24^{\prime \prime} \mathrm{E}\right)$. Algae collected from seagrass bed, dead staghorn corals and isolated limestone rocks, 0.5-5.0 m, 28 May 1978.

78 EM-23: Actin, Basay, Negros Oriental $\left(9^{\circ} 25^{\prime} 00^{\prime \prime} \mathrm{N}\right.$, $\left.122^{\circ} 36^{\prime} 12^{\prime \prime} \mathrm{E}\right)$. Algae collected from Sargassum bed, rocks and stony corals, 0.5-5.0 m, 29 May 1978.

78 EM-24: Cabcab, Zamboanguita, Negros Oriental $\left(9^{\circ} 05^{\prime} 30^{\prime \prime} \mathrm{N}, 123^{\circ} 11^{\prime} 15^{\prime \prime} \mathrm{E}\right)$. Algae collected from seagrass bed and back reef, 0.2-5.0 m, 2 June 1978 .

78 EM-25: Banilad, Dumaguete City, Negros Oriental $\left(9^{\circ} 16^{\prime} 45^{\prime \prime} \mathrm{N}, 123^{\circ} 18^{\prime} 15^{\prime \prime} \mathrm{E}\right)$. Algae collected from isolated rocks, seagrass bed and back reef, 0.5-5.0 m, 3 June 1978.

78 EM-26: Tapon-Norte, San Jose, Negros Oriental 
$\left(9^{\circ} 25^{\prime} 00^{\prime \prime} \mathrm{N}, 123^{\circ} 14^{\prime} 15^{\prime \prime} \mathrm{E}\right)$. Algae collected from rocky outcrops, seagrass bed and corals, 0.5-4.0 m, 5 June 1978.

78 EM-27: Suyac Island, off old town of Sagay, Negros Occidental $\left(10^{\circ} 57^{\prime} 15^{\prime \prime} \mathrm{N}, 123^{\circ} 27^{\prime} 00^{\prime \prime} \mathrm{E}\right)$. Algae collected from coral rubbles, 0.3-6.0 m, 8 June 1978 .

78 EM-28: Rocky area between Ilacaon Island and Cadiz, Negros Occidental $\left(11^{\circ} 00^{\prime} 45^{\prime \prime} \mathrm{N}, 123^{\circ} 15^{\prime} 52^{\prime \prime} \mathrm{E}\right)$. Algae collected from rocks, $2.0 \mathrm{~m}, 9$ June 1978.

78 EM-29: Ilacaon Island, Negros Occidental $\left(11^{\circ} 02^{\prime} 22^{\prime \prime} \mathrm{N}\right.$, $\left.123^{\circ} 12^{\prime} 00^{\prime \prime} \mathrm{E}\right)$. The substrate consists of rocks, corals and seagrass, 0.3-6.0 m, 9 June 1978.

78 EM-30: Sumilon Island $\left(9^{\circ} 27^{\prime} 00^{\prime \prime} \mathrm{N}, 123^{\circ} 23^{\prime} 24^{\prime \prime} \mathrm{E}\right)$. Algae collected from limestone rocks and corals, $0.3-6.0 \mathrm{~m}$, 15 June 1978.

78 EM-31: Ayungon, Negros Oriental $\left(9^{\circ} 50^{\prime} 30^{\prime \prime} \mathrm{N}, 123^{\circ}-\right.$ $\left.08^{\prime} 30^{\prime \prime} \mathrm{E}\right)$. Rocky platform, seagrass and coral rubbles, 0.3-3.0 m, 17 June 1978.

78 EM-32: Apo Island $\left(9^{\circ} 04^{\prime} 55^{\prime \prime} \mathrm{N}, 123^{\circ} 16^{\prime} 30^{\prime \prime} \mathrm{E}\right)$. Algae on limestone rocks and dead staghorn corals, 0.5-3.0 m, 22 June 1978.

78 EM-33: Apo Island, northern end $\left(9^{\circ} 05^{\prime} 09^{\prime \prime} \mathrm{N}, 123^{\circ}\right.$ $\left.16^{\prime} 00^{\prime \prime} \mathrm{E}\right)$. Rocks and stony corals provide excellent substrate for algae, $0.4-3.0 \mathrm{~m}, 23$ June 1978.

78 EM-34: Apo Island, western end $\left(9^{\circ} 04^{\prime} 40^{\prime \prime} \mathrm{N}, 123^{\circ}\right.$ $\left.16^{\prime} 00^{\prime \prime} \mathrm{E}\right)$. Algae collected from limestone rocks and corals, 0.5-3.0 m, 23 June 1978.

78 EM-37: Bantayan Beach, Dumaguete City, Negros Oriental $\left(9^{\circ} 22^{\prime} 30^{\prime \prime} N, 123^{\circ} 15^{\prime} 45^{\prime \prime} \mathrm{E}\right)$. Seagrasses and corals provide good substrate for a rich algal flora, 1.0-2.0 m, 23 June 1978.

78 EM-39: Calindagan Reef, off Dumaguete City, Negros Oriental $\left(9^{\circ} 21^{\prime} 00^{\prime \prime} \mathrm{N}, 123^{\circ} 16^{\prime} 00^{\prime \prime} \mathrm{E}\right)$. Algae collected by local diver using SCUBA, $26 \mathrm{~m}, 6$ June 1978.

78 EM-40: Bacolod City, Negros Occidental $\left(10^{\circ} 41^{\prime} 15^{\prime \prime} \mathrm{N}\right.$, $122^{\circ} 56^{\prime} 45^{\prime \prime} \mathrm{E}$ ). Algae collected nearshore from sand substrate, 8 June 1978.

79 EM-8: Pescador Island, Moalboal, Cebu $\left(9^{\circ} 55^{\prime} 30^{\prime \prime} \mathrm{N}\right.$, $123^{\circ} 20^{\prime} 36^{\prime \prime} \mathrm{E}$ ). Few algae collected from crevices on rocks and corals, 0.3-4.5 m, 7 May 1979.

79 EM-9: Pinagsamahan Beach, Moalboal, Cebu $\left(9^{\circ}\right.$. $56^{\prime} 12^{\prime \prime} \mathrm{N}, 123^{\circ} 23^{\prime} 25^{\prime \prime} \mathrm{E}$ ). Algae abundant in seagrass bed and corals, $1.0-4.5 \mathrm{~m}, 8$ May 1979 .

79 EM-11: Northern end of Apo Island, Dauin, Negros Oriental $\left(9^{\circ} 05^{\prime} 20^{\prime \prime} \mathrm{N}, 123^{\circ} 16^{\prime} 10^{\prime \prime} \mathrm{E}\right)$. Fore-reef slope with few algae, $12.0-36.5 \mathrm{~m}, 10$ May 1979

79 EM-12: Northwestern portion of Apo Island, Dauin,
Negros Oriental $\left(9^{\circ} 04^{\prime} 45^{\prime \prime} \mathrm{N}, 123^{\circ} 16^{\prime} 00^{\prime \prime} \mathrm{E}\right)$. Algae sparse on rocks and rubbles, 0.5-1.5 m, 10 May 1979.

79 EM-13: Southern end of Apo Island, Dauin, Negros Oriental $\left(9^{\circ} 04^{\prime} 10^{\prime \prime} \mathrm{N}, 123^{\circ} 16^{\prime} 00^{\prime \prime} \mathrm{E}\right)$. Algae collected from rocks exposed to heavy surf, 0.3-3.5 m, 11 May 1979.

79 EM-14: Southeastern portion of Apo Island, Dauin, Negros Oriental $\left(9^{\circ} 04^{\prime} 30^{\prime \prime} \mathrm{N}, 123^{\circ} 16^{\prime} 15^{\prime \prime} \mathrm{E}\right)$. Algae collected from staghorn corals, 0.6-4.5 m, 11 May 1979.

79 EM-15: Malo, Siaton, Negros Oriental $\left(9^{\circ} 03^{\prime} 00^{\prime \prime} \mathrm{N}\right.$, $\left.122^{\circ} 59^{\prime} 00^{\prime \prime} \mathrm{E}\right)$. Algae attached to rocks at $3.0 \mathrm{~m}, 14 \mathrm{May}$ 1979 .

79 EM-16: Polopantao, Jimalalud, Negros Oriental $\left(9^{\circ}\right.$. $59^{\prime} 00^{\prime \prime} \mathrm{N}, 123^{\circ} 12^{\prime} 06^{\prime \prime} \mathrm{E}$ ). Algae attached to rocks and corals near river mouth, 1.0-3.0 m, 15 May 1979.

79 EM-18: Barrio Dumanhog, northwestern side of Siquijor Island $\left(9^{\circ} 12^{\prime} 57^{\prime \prime} \mathrm{N}, 123^{\circ} 30^{\prime} 14^{\prime \prime} \mathrm{E}\right)$. Algae depauperate in seagrass bed and rubbles, $4.5 \mathrm{~m}, 14$ May 1979.

79 EM-21: Barrio Dapdap, northwestern side of Siquijor Island $\left(9^{\circ} 13^{\prime} 21^{\prime \prime} \mathrm{N}, 123^{\circ} 29^{\prime} 05^{\prime \prime} \mathrm{E}\right)$. Few algae collected from rocks, $4.5 \mathrm{~m}, 15$ May 1979.

79 EM-22: Barrio Cang-aluang, northwestern side of Siquijor Island $\left(9^{\circ} 13^{\prime} 24^{\prime \prime} \mathrm{N}, 123^{\circ} 28^{\prime} 48^{\prime \prime} \mathrm{E}\right)$. Algae collected from coral reef, $4.5 \mathrm{~m}, 15$ May 1979.

79 EM-23: Barrio Sabang, Larena, northwestern side of Siquijor Island $\left(9^{\circ} 14^{\prime} 27^{\prime \prime} \mathrm{N}, 123^{\circ} 34^{\prime} 00^{\prime \prime} \mathrm{E}\right)$. Algae collected from coral reef, $4.5 \mathrm{~m}, 16$ May 1979.

\section{Class Chlorophyceae}

\section{Family Caulerpaceae}

\section{Genus Caulerpa}

Caulerpa Lamouroux, 1809a:136

Thallus a branched coenocyte, differentiated into a cylindrical, prostrate, creeping stolon and erect assimilative branches; attached by branched rhizoids; erect branches simple or branched, usually bearing terete, flat or compressed branchlets. Thallus internally traversed by extensions (trabeculae) of the cell wall. Reproduction sexual or asexual (anisogamous), when sexual, biflagellated gametes are liberated through branch papillae. Plants are widely distributed in tropical and subtropical seas.

\section{Key to Species of Caulerpa from Central Visayas}

1. Erect branches angular, with thorn-like or dentate projections, stolons naked

C. urvilliana

Erect branches not angular, stolons naked or tomentose 
2. Stolons and branches similar in form ...................... 3

Stolons and branches dissimilar in form .................... 4

3. Erect branches bearing verticillate branchlets .......... verticillata

Erect branches without verticillate branchlets .......... C. fastigiata

4. Erect branches flat or compressed ...................... 5

Erect branches not flat or compressed ................... 10

5. Ramelli present ................................... 6

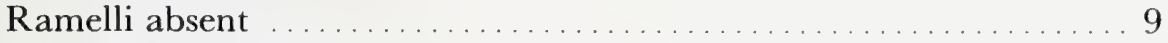

6. Ramelli cylindrical ............................... 7

Ramelli flat or compressed ......................... 8

7. Plants small, to $15 \mathrm{~mm}$ tall, branches substipitate, bearing pinnules to

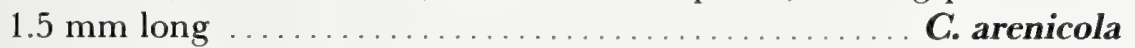

Plants moderately large, to $60 \mathrm{~mm}$ tall, branches long-stalked, bearing pinnules to $8 \mathrm{~mm}$ long ...................... sertularioides

8. Branchlets oppositely-pinnate; stolons $200 \mu \mathrm{m}$ in diameter

C. crassifolia

Branchlets tetrastichous, distichous; stolons 1-2 $\mathrm{mm}$ in diameter

C. reyesii, new species

9. Branches dichotomous, margins regularly serrate ....... C. serrulata

Branches proliferous, margins irregularly serrate ....... C. brachypus

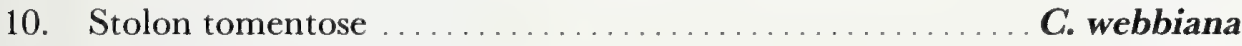

Stolon naked ..................................... 11

11. Ramelli seminaviculate, in several ranks ............ cupressoides

Ramelli clavate or abruptly ending in swollen tips $\ldots \ldots \ldots \ldots \ldots 12$

12. Ramelli thick-walled, not translucent ............... C. racemosa

Ramelli thin-walled, translucent ....................... 13

13. Ramelli many, imbricate or in 4-rows; branches long and near each other

C. lentillifera

Ramelli few; branches short, distantly spaced …...... microphysa

* Caulerpa arenicola Taylor

Plate 1h

Caulerpa arenicola Taylor, 1950:55, 204, pl. 28: fig. 2.

Description.-Plants small, forming loose mats on sand, to $15 \mathrm{~mm}$ tall; the creeping stolon branched, terete, to $0.5 \mathrm{~mm}$ in diameter, bearing very short, slender, few descending branches with filiform rhizoids at the ends; the substipitate, erect foliar branches simple or forked once or twice, with terete rachis, to $300 \mu \mathrm{m}$ in diameter, bearing oppositely-pinnate, cylindrical, filiform pinnules, $250-300 \mu \mathrm{m}$ in diameter, to $1.5 \mathrm{~mm}$ long, slightly upcurved to straight, distal end conical, apiculate with basal end somewhat nar- rower; the foliar branches of even margin, or interrupted occasionally by shorter pinnules.

Specimens Studied.-78 EM-9(126-130, 178); 78 EM-25(7); 79 EM-16(50).

Philippine Distribution.-Visayas (Siquijor, Negros Oriental).

\section{Caulerpa brachypus Harvey}

Plate 1

Caulerpa brachypus Harvey, 1860:333._Gilbert, 1961:435.Domantay, 1962:279._Taylor, 1966:350; 1977:4.Trono, 1972:95; 1973:217.-Cordero, 1974:6; 1977:25, figs. 2, 3.-Reyes, 1976:153, pl. 7: fig. 4.

Description.-Plants of moderate size, form- 
SMITHSONIAN CONTRIBUTIONS TO THE MARINE SCIENCES

ing dense, long and spreading mats on sand-mud substrate among seagrasses, to $50 \mathrm{~mm}$ tall; the prostrate, sparingly branched, terete stolon to 1 $\mathrm{mm}$ in diameter, with few descending branches bearing filiform rhizoids at the ends; the substipitate, erect foliar branches proliferous occasionally, ligulate, 5-40 $\mathrm{mm}$ long, 2-4 $\mathrm{mm}$ wide, with serrate margins or almost entire in a few specimens, irregularly disposed, $2-15 \mathrm{~mm}$ distance apart on upper side of stolon.

Specimens Studied.-78 EM-5(29-33， 298316); 79 EM-22(1, 2).

Philippine Distribution.-Luzon (Pangasinan); Visayas (Siquijor, Negros Oriental, Negros Occidental); Mindanao (Zamboanga, Basilan, Sulu).

\section{** Caulerpa crassifolia (C. Agardh) J. Agardh}

Plate 1D,E

Caulerpa taxifolia var. crassifolia C. Agardh, 1823:436.

Caulerpa crassifolia (C. Agardh) J. Agardh, 1873:13.-Gilbert, 1942:12; 1946:78; 1961:436.-Meñez, 1961:53.

DESCRIPTION.-Plants forming loose clusters on sand, at $10 \mathrm{~m}$, to $43 \mathrm{~mm}$ tall; the creeping stolon terete, slender, no more than $200 \mu \mathrm{m}$ in diameter, bearing descending branches with filiform, laxly branched rhizoids at the ends; the stipitate, erect foliar branches simple, with compressed rachis, 1-1.5 mm wide, bearing oppositely-pinnate, flat to compressed ramelli, $0.5-1 \mathrm{~mm}$ wide, $1.5-3.5$ $\mathrm{mm}$ long, almost with an even width, except for slight attenuation near the rounded tip, longer ramelli slightly curved; ramelli at mid-portion of foliar branch longer than basal and distal portions; foliar branches very few, distantly spaced on upper side of prostrate stolon, with stipes rarely exceeding $5 \mathrm{~mm}$ in length.

Specimens Studied.-HPG-6 (material collected by H.P. Calumpong).

Philippine Distribution.-Luzon (Pangasinan); Visayas (Negros Oriental).

Remarks.-Specimens were collected from sandy bottom, $10 \mathrm{~m}$ deep, from Ajong, Sibulan, Negros Oriental on 12 November 1979. Plants examined are smaller compared to those described by Gilbert (1942:12) from West Java.

\section{Caulerpa cupressoides (West in Vahl) C. Agardh \\ Plate 1B,C}

Fucus cupressoides West in Vahl, 1802:38.

Caulerpa cupressoides (West in Vahl) C. Agardh, 1823:441.Reyes, 1972:142; 1976:155, pl. 8: fig. 7.

Description.-Plants of moderate size, forming loose mats on rocks and seagrasses, to $60 \mathrm{~mm}$ tall; the creeping, terete, naked, sparingly branched stolon to $2 \mathrm{~mm}$ in diameter, bearing descending branches with thick, branched, filiform rhizoids at the ends; the stipitate, erect, foliar branches simple or branched, with terete or slightly compressed rachis, to $1.5 \mathrm{~mm}$ in diameter in larger plants, bearing multiseriate or occasionally pinnate, upcurved, compressed, seminaviculate, ovoid, conical, sometines linear branchlets, to $2 \mathrm{~mm}$ long, not exceeding $0.5 \mathrm{~mm}$ at the widest portion, with cuspidate or mucronate tips.

SPEcimens Studied._-78 EM-3(62-65); 78 EM-5(243-245); 78 EM-9(131, 179, 243); 78 EM18(75-79);78 EM-25(8-11).

Philippine Distribution.-Visayas (Negros Oriental, Siquijor).

\section{Caulerpa fastigiata Montagne}

Plate 1a

Caulerpa fastigiata Montagne, 1837:353.—Gilbert, 1942:9; 1961:436.—-Reyes, 1976:154, pl. 8: figs. 1, 2.

Description.-Plants filiform, forming thick mats on rocks and sand, to $30 \mathrm{~mm}$ tall; the terete, creeping stolon to $300 \mu \mathrm{m}$ in diameter, with laxly branched, filiform rhizoids borne at the ends of descending filaments; the ascending filaments morphologically similar to stolons, with subopposite, alternate, dichotomous or occasionally subverticillate branches, to $1 \mathrm{~mm}$ long, 150-225 $\mu \mathrm{m}$ in diameter, with obtuse or rounded apices.

SPecimens Studied.-78 EM-5(215-225); 78 
EM-27(29A); 78 EM-31(25); 78 EM-34(643); 78 EM-37(16).

Philippine Distribution.-Luzon (Oriental Mindoro); Visayas (Negros Oriental, Siquijor, Suyac, Apo); Mindanao (Zamboanga, Basilan).

\section{Caulerpa lentillifera J. Agardh}

$$
\text { Plate 1F, G }
$$

Caulerpa lentillifera J. Agardh, 1837:173.-Gilbert, 1942:23, 1961:436. - Domantay, 1962:277.-Trono, 1972:96; 1973: 217; 1975:33; 1978:4.-Reyes, 1976:155, 162, pl. 8: fig. 6.-Trono and Tuazon, 1978:3.

Description.-Plants small, in loose mats on coral rubbles and rocks, to $30 \mathrm{~mm}$ tall; the terete stolon widespread, to $2 \mathrm{~mm}$ in diameter, bearing long, descending branches, to $20 \mathrm{~mm}$ long, with laxly branched, filiform rhizoids at the ends; the few, shortly stipitate, erect branches simple or subsimple, with terete rachis, $1-1.5 \mathrm{~mm}$ in diameter, bearing spherical translucent ramelli, to $1.5 \mathrm{~mm}$ in diameter, supported by stalks, $0.5-1.0$ $\mathrm{mm}$ long; ramelli conspicuously constricted at the base, imbricately arranged or in rows of four.

Specimens Studied.-78 EM-5(34, 227, 228); 79 EM-18(62, 63); 79 EM-23(12).

Philippine Distribution.-Luzon (Oriental Mindoro, Pangasinan, Sorsogon, Catanduanes, Marinduque); Visayas (Negros Oriental, Siquijor); Mindanao (Zamboanga, Basilan, Sulu).

\section{** Caulerpa microphysa (Weber-van Bosse) Feldmann}

\section{Plate 2L}

Caulerpa racemosa var. clavifera $\mathrm{f}$. microphysa Weber-van Bosse, 1898:361, pl. 33: fig. 5.

Caulerpa microphysa (Weber-van Bosse) Feldmann, 1955: 430.-Taylor, 1966: 350.

Description.-Plants small, forming dense clusters on corals and rocks, to $15 \mathrm{~mm}$ tall; the prostrate, terete, naked stolon less than $1 \mathrm{~mm}$ in diameter, bearing descending branches with branched, filiform rhizoids at the ends; the ascending branches on the upper side of the stolon shortly stipitate, simple or occasionally branched, with terete rachis bearing crowded, spherical ramelli, $1-1.5 \mathrm{~mm}$ in diameter, supported by stalks, $0.5 \mathrm{~mm}$ long, constricted at the apical end.

Specimens Studied.-78 EM-5(34A); 78 EM33(545-547); 78 EM-30(10-12); 78 EM-34(137139); 79 EM-8(10-15); 79 EM-11(11); 79 EM$12(7-9,105-107) ; 79$ EM-18(63-67); 79 EM21(16,17); 79 EM-22(15-17).

Philippine Distribution.-Visayas (Negros Oriental, Sumilon, Apo, Pescador, Siquijor); Mindanao (Zamboanga).

\section{Caulerpa racemosa (Forsskål) J. Agardh}

Fucus racemosus Forsskål, 1775:191.

Caulerpa racemosa (Forsskål). J. Agardh, 1873:35.--Gilbert, 1946: 78.-Velasquez, 1953:100.-Meñez, 1961:51.-Taylor, 1966:350._Reyes, 1972:142.-Trono, 1972:96; 1973: 218.-Velasquez, Cornejo, Santiago, and Baens-Arcega, 1973:14, pl. 4: fig. 20.--Trono, 1975:33; 1978:5._Velasquez, Trono, and Doty, 1975:131.-Trono and Tuazon, 1978:3.-Cordero, 1978:280.

DESCRIPTION.-Plants highly variable, forming loose to dense, sometimes entangled colonies, consisting of few to many erect branches, simple or branched, bearing clavate, turbinate, spherical, hemispherical or discoid ramelli, which are stipitate or substipitate, having obtuse or flattened tips; ramelli distichously, multiseriately or imbricately arranged on terete rachis; creeping stolon terete, with short or long descending branches, giving off branched rhizoids at the ends.

\section{** Caulerpa racemosa var. clavifera (Turner) Weber-van Bosse}

$$
\text { Plate 2A }
$$

Fucus clavifer Turner, 1808:126.

Caulerpa clavifera (Turner) C. Agardh, 1817:xxiii.-Dickie, 1877:244--Howe, 1932:169.

Caulerpa racemosa var. clavifera (Turner) Weber-van Bosse, 1898:361.-Gilbert, 1946:78; 1961:437.-Galutira and Velasquez, 1964:496.

Description.-Plants up to $50 \mathrm{~mm}$ tall, with irregularly spaced erect branches, simple or oc- 
casionally branched, bearing ramelli that are gradually expanded into a round end, to $2 \mathrm{~mm}$ in diameter; ramelli distichously or irregularly arranged on terete rachis.

Specimens Studied.-78 EM-32(53); 78 EM33(548-552); 79 EM-12(13-18); 79 EM-23(11, 12).

Philippine Distribution.-Luzon (Batanes, Babuyan Island, La Union, Ilocos Norte, Pangasinan, Bataan, Batangas, Quezon, Oriental Mindoro); Visayas (Panay, Negros Oriental, Apo); Mindanao (Zamboanga, Misamis Oriental, Basilan, Sulu).

\section{Caulerpa racemosa var. macrophysa (Kützing)} Taylor

Plate 2c

Chauvinia macrophysa Kützing, 1857:6.

Caulerpa racemosa var. macrophysa (Kützing) Taylor, 1928: 101.-Gilbert, 1942:19; 1961:439.-Domantay, 1962: 277.-Taylor, 1966:351.

Description.-Plants large, to $100 \mathrm{~mm}$ tall; erect branches simple or occasionally branched, few and distantly spaced on stout, creeping stolon; branches bearing ramelli expanded into a hemispherical end and arranged irregularly on terete rachis.

Specimens Studied.-78 EM-5(1-23, 260-263, 270-297); 79 EM-18(68-71, 80, 81); 79 EM-21(611); 79 EM-22(64).

Philippine Distribution.-Luzon (Pangasinan, Oriental Mindoro); Visayas (Cebu, Negros Oriental, Siquijor); Mindanao (Basilan, Sulu).

** Caulerpa racemosa var. occidentalis (J. Agardh) Børgesen

Plate 2B

Caulerpa chemnitzia var. occidentalis J. Agardh, 1873:37.

Caulerpa racemosa var. occidentalis (J. Agardh) Børgesen, 1907:379._Domantay, 1962:278. - Taylor, 1966:351.

Description.-Plants up to $50 \mathrm{~mm}$ tall; creeping, terete stolon giving off erect branches, simple or rarely branched, bearing ramelli abruptly ex- panded into subspherical ends, reaching $4 \mathrm{~mm}$ in diameter, distichously or radially arranged on terete rachis.

Specimens Studied.-78 EM-1(13, 14, 50, 51, 77, 78); 78 EM-9(132); 78 EM-10(80, 81); 78 EM-23(183); 78 EM-24(2-5); 78 EM-26(1, 2); 78 EM-28(53); 78 EM-29(24-29); 78 EM-30(1-5); 78 EM-31 (1, 2); 78 EM-34(131); 78 EM-37 (15);78 EM-38(383, 390, 392, 394); 79 EM-8(17); 79 EM-12(13-18, 108-117); 79 EM-14(6-12); 79 EM-21(5).

Philippine Distribution.-Luzon (Oriental Mindoro, Pangasinan, Sorsogon, Catanduanes, Marinduque); Visayas (Negros Oriental, Siquijor); Mindanao (Zamboanga, Basilan, Sulu).

\section{** Caulerpa racemosa var. peltata (Lamouroux) Eubank}

\section{Plate 2k}

Caulerpa peltata Lamouroux, 1809b:332.—Gilbert, 1942:22.

Caulerpa racemosa var. peltata (Lamouroux) Eubank, 1946 421.-Gilbert, 1961:439.

Description.-Specimens belonging to this species exhibit several different forms, but consistently show the characteristic of having peltate disks at the distal ends of short stalks. The same plant may bear peltate and spherical to turbinate ramelli. The discoid ramelli may be borne on ascending branches, arranged alternately in several ranks or produced directly from the dorsal side of the creeping stolon and supported by terete stipes. Plants found in sheltered areas seem to be the largest, having terete stolons, to $1 \mathrm{~mm}$ in diameter, bearing single, stipitate, peltate disks, 1-5 $\mathrm{mm}$ in diameter. The depauperate forms were mostly found in rocky, exposed areas and these showed a high degree of polymorphism. They were smaller; slender stolon to $300 \mu \mathrm{m}$ in diameter, bearing single, stipitate, peltate disks, which are less than $2 \mathrm{~mm}$ in diameter; disks sometimes several on erect branches; from margins of disks, occasionally other branches are produced. The stolon bears short, descending branches, with branched filiform rhizoids at the ends. 
Specimens Studied.-78 EM-25(1-6); 78 EM33(384, 386-389, 397-399, 401, 402, 542-544); 78 EM-34(124-130, 132, 140-145); 78 EM-30(6); 78 EM-32(24); 79 EM-8(16); 79 EM-11(16, 17); 79 EM-12(10-12, 118-120); 79 EM-15(311); 79 EM18(59-61); 79 EM-21(9-12); 79 EM-22(11-14).

Philippine Distribution.-Luzon (Oriental Mindoro); Visayas (Negros Oriental, Siquijor, Apo, Sumilon, Pescador); Mindanao (Zamboanga).

\section{** Caulerpa racemosa var. uvifera (Turner)} J. Agardh

Plate 2D

Fucus uvifer Turner, 1819:81, pl. 230.

Caulerpa racemosa var. uvifera (Turner) J. Agardh, 1873:35.Gilbert, 1961:440.

Description.-Plants simple, erect branches to $30 \mathrm{~mm}$ tall, bearing stalked ramelli, imbricately disposed, closely crowded, gradually expanded to swollen tips; length of stalk supporting ramelli about the same as diameter of swollen ends.

Specimens Studied._- 79 EM-22(60-63).

Philippine Distribution.-Visayas (Siquijor); Mindanao (Zamboanga).

\section{Caulerpa serrulata (Forsskål) J. Agardh}

\section{Plate 2E}

Fucus serrulatus Forsskål, 1775:189.

Caulerpa serrulata (Forsskål) J. Agardh, 1837:174.—Gilbert, 1942:14; 1946:78; 1961:440.—Domantay, 1962:278. Meñez, 1961:53._Taylor, 1966:351.—Reyes, 1972:142._ Trono, 1972:96; 1973:218; 1975:33.-Cordero, 1973:19.Velasquez, Cornejo, Santiago, and Baens-Arcega, 1973:15, pl. 5: fig. 21.-Trono and Tuazon, 1978:3.

Description.-Plants moderately large, widespread, in dense colonies on rocks and coral rubbles, to $50 \mathrm{~mm}$ tall; the creeping, terete stolon with few, short, descending branches having branched rhizoids at the ends; ascending branches many, flat or compressed, twisted, once to several times dichotomously or irregularly branched; branches regularly with serrate mar- gins, to $30 \mathrm{~mm}$ long, $3 \mathrm{~mm}$ broad, supported by terete stalks, 3-7 mm long.

Specimens Studied.-78 EM-7(1); 78 EM9(114-120); 78 EM-25(21); 78 EM-29(6-10); 78 EM-30(11); 78 EM-31(4-9); 79 EM-8(6-9); 79 EM-9(18); 79 EM-15(197-200); 79 EM-16(48, 49).

Philippine Distribution.-Luzon (Ilocos Sur, Pangasinan, Bataan, Batangas, Catanduanes, Sorsogon, Marinduque, Oriental Mindoro); Visayas (Leyte, Siquijor, Sumilon, Apo, Negros Oriental); Mindanao (Zamboanga, Basilan).

\section{** Caulerpa serrulata var. boryana f. occidentalis (Weber-van Bosse) \\ Yamada and Tanaka}

$$
\text { Plate 2F }
$$

Caulerpa freycinetii var. boryana f. occidentalis Weber-van Bosse, 1898:315, pl. 25: fig. 11.

Caulerpa serrulata var. boryana f. occidentalis (Weber-van Bosse) Yamada and Tanaka, 1938:62.-Gilbert, 1942:15; 1961:441.

Description.-Erect branches to $30 \mathrm{~mm}$ tall, 1-3 times dichotomously branched, flat or compressed, supported by a long, terete stalk, to 7 mm long; branches not twisted.

Specimen Studied.-78 EM-24(1).

Philippine Distribution.-Palawan (Culion); Visayas (Negros Oriental); Mindanao (Sulu).

\section{Caulerpa sertularioides (Gmelin) Howe}

$$
\text { Plate 3F }
$$

Fucus seriularioides Gmelin, 1768:151.

Caulerpa sertularioides (Gmelin) Howe, 1905:576._Blanco, 1938:512.—Gilbert, 1946:78; 1961:441.-Domantay, 1962:278.-Meñez, 1961:52.-Taylor, 1966:351.--Reyes, 1972:143.-Velasquez, Cornejo, Santiago, and Baens-Arcega, 1973:14, pl. 4: fig. 19.-Trono, 1972:96.-Cordero, 1978:280.

Description.-Plants moderately large, widespread, forming loose colonies on rocks and corals, to $60 \mathrm{~mm}$ (rarely $100 \mathrm{~mm}$ ) tall; stolon terete, to $1 \mathrm{~mm}$ in diameter, bearing few, short, descending branches with branched rhizoids at the ends; the 
erect, simple or occasionally branched, featherlike branches with long stalks extending into a terete rachis, to $1 \mathrm{~mm}$ in diameter, bearing cylindrical, pinnately arranged pinnules, commonly 8 $\mathrm{mm}$ long, $200 \mu \mathrm{m}$ in diameter, upcurved with mucronate tips.

Specimens Studied.-78 EM-5(35-38, 317323); 78 EM-22(2, 3, 14); 78 EM-40(1); 79 EM18(72-74); 79 EM-23(9, 10).

Philippine Distribution.-Luzon (Babuyan Island, Pangasinan, Bataan, Batangas, Manila Bay, Oriental Mindoro, Sorsogon); Visayas (Siquijor, Aklan, Negros Oriental, Negros Occidental); Mindanao (Sulu).

\section{Caulerpa urvilliana Montagne}

Plate 3D,e

Caulerpa urvilliana Montagne, 1845:21.-Taylor, 1966:352.Trono, 1972:97; 1973:219.--Reyes, 1976:154, pl. 7: fig. 5.

Description.-Plants large, commonly forming loose colonies on sand, to $150 \mathrm{~mm}$ tall; the terete stolon to $3.5 \mathrm{~mm}$ in diameter, bearing few to many cylindrical, descending branches with long, branched rhizoids; the terete, erect branches several times dichotomously or alternately branched, with mamillate, multiseriately disposed protuberances and mucronate tips; these erect branches, $20-140 \mathrm{~mm}$ tall, supported by terete stalk, 10-60 $\mathrm{mm}$ long.

Specimens Studied.-78 EM-5(39-46, 246259); 79 EM-21(13-15); 79 EM-22(56-59, 95).

Philippine Distribution.-Luzon (Sorsogon); Palawan; Visayas (Siquijor, Negros Oriental, Negros Occidental); Mindanao.

\section{* Caulerpa webbiana Montagne}

$$
\text { Plate 2G-J }
$$

Caulerpa webbiana Montagne, 1838:129.

Description.-Plants small, in thick cushions on exposed rocks, to $15 \mathrm{~mm}$ tall; the terete, creeping stolon beset with few, short, determinate branchlets, divided once to few times; the terete, descending branches on the ventral side of the stolon, naked or with few determinate branchlets, bearing laxly branched rhizoids at the ends; ascending branches from stolon many, simple or branched, 10-15 mm tall, having many whorls, sometimes distichous determinate branchlets 1-5 times divided, with mucronate tips. Plants in sheltered habitat have fewer, but longer branches, occasionally with distichous determinate branchlets.

Specimens Studied.-78 EM-32(54); 78 EM33(132-144, 541).

Philippine Distribution.-Visayas (Apo).

\section{Caulerpa verticillata J. Agardh}

$$
\text { Plate 3A-C }
$$

Caulerpa verticillata J. Agardh, 1847:6.—Gilbert, 1961:442._ Trono, 1973:219._Reyes, 1976:154, pl. 8: fig. 3.

Description.-Plants filiform, attached to rocks and corals, to $50 \mathrm{~mm}$ tall; the creeping, naked terete stolon with numerous short, descending branches, terminated by laxly branched, filiform rhizoids; the terete, erect branches, simple or irregularly branched, bearing abundant tufts of whorled, determinate branchlets, to $180 \mu \mathrm{m}$ in diameter, to 8 times dichotomously branched; the filiform branchlets constricted at the base, with 2-3 teeth at the apices.

Specimens Studied.-79 EM-12(3-6).

Philippine Distribution.-Palawan; Visayas (Siquijor, Apo); Mindanao (Sulu, Basilan).

\section{* Caulerpa reyesii, new species}

\section{Figure 2; Plate 3G-I}

Description.-Plantae mediocriter robustae in coloniis laxis late effusae. Stolones repentes teretes ramosi ad $2.5 \mathrm{~mm}$ in diametro; rami descendentes ad $60 \mathrm{~mm}$ longi in apicem rhizoideis filiformis ferentes; rami ascendentes $10-70 \mathrm{~mm}$ alti simplices vel interdum frondiformes; frondes basi vel ad medio ramosae basi breviter terete stipitatae, stipitis in rachidis compressis $1 \mathrm{~mm}$ latis continuis, ramulis subsessilibus complanatis obovatis 


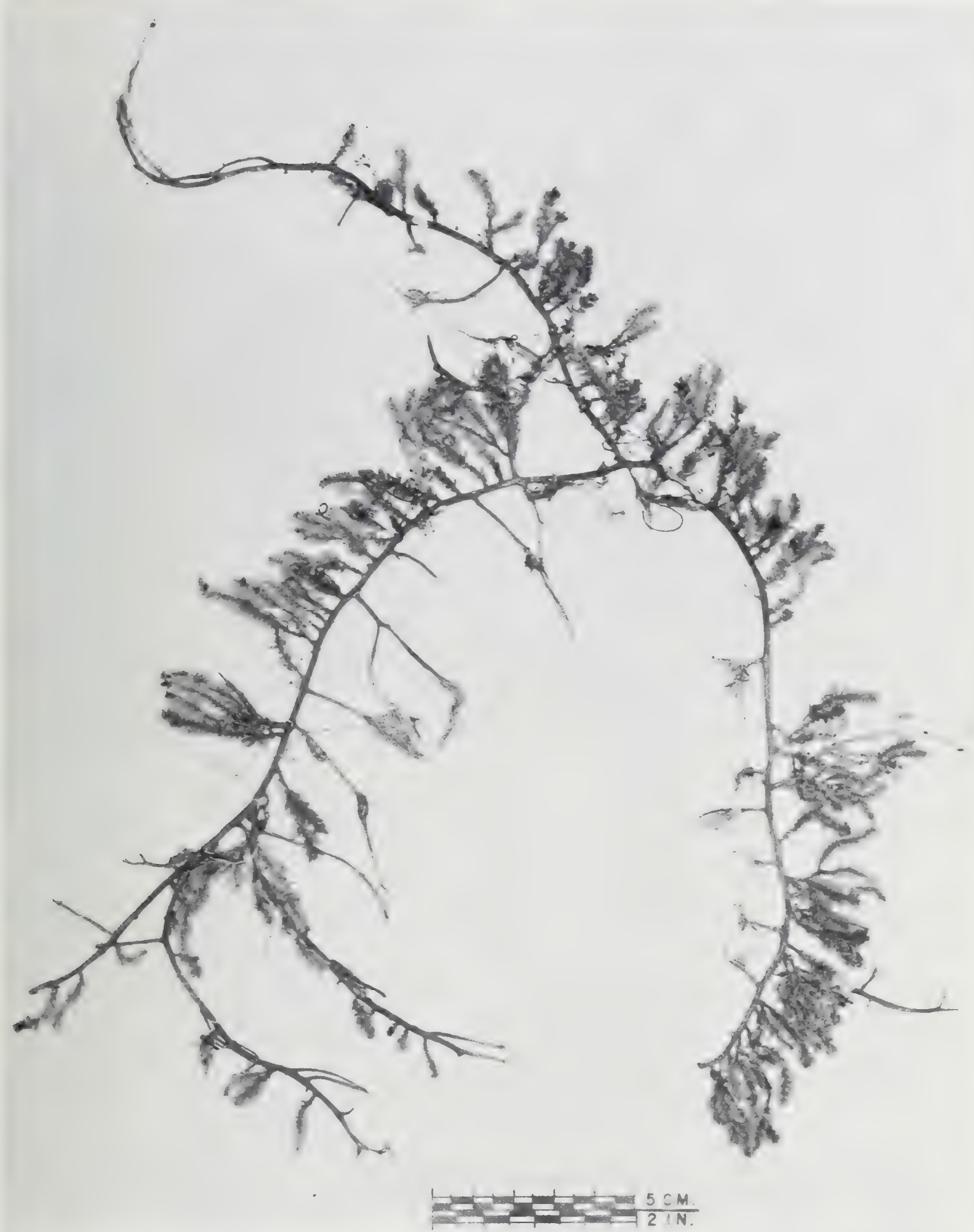

Figure 2.- Habit of Caulerpa reyesii, new species. Holotype: 78 EM-5(330), Solong-on, Siquijor Island, 10 May 1978. 
clavatis adaxialiter incurvalis ad $7 \mathrm{~mm}$ longis et in partibus latissimus $2 \mathrm{~mm}$ latis plerumque as apicem mucronatis vel cuspidatis distichis vel variabiliter tetrastichis et subimbricatis.

Plants moderately robust, widespread, forming loose colonies. The creeping stolon terete, branched, to $2.5 \mathrm{~mm}$ in diameter, bearing long, terete, descending branches, to $60 \mathrm{~mm}$ long, with filiform rhizoids at the ends. The ascending branches from stolon, $10-70 \mathrm{~mm}$ tall, simple or if branched, secondary branching produced from the mid-portion or the lower margin of the compressed rachis; erect branches supported by short, cylindrical stalks that extend into a compressed rachis, $1 \mathrm{~mm}$ broad, bearing subsessile, flat, overlapping, obovate, clavate ramelli, which are adaxially incurved, tetrastichous, distichous, to $7 \mathrm{~mm}$ long and $2 \mathrm{~mm}$ at the widest portion, mostly mucronate or cuspidate at the tips.
Holotype. - 78 EM-5(330), on coral rubbles and sand in seagrass bed, at Solong-on, Siquijor Island, Philippines, 10 May 1978; deposited in the U.S. National Herbarium, Smithsonian Institution, Washington, D.C., U.S.A. Isotypes deposited in the Philippine National Herbarium, Manila, Philippines and Silliman University Herbarium, Dumaguete City, Philippines.

Paratypes Studied.-78 EM-5(24-28).

Remarks.-It is with great pleasure to name this species of Caulerpa after our colleague and friend, Professor Alfredo Reyes, Department of Biology, Silliman University, Dumaguete City, Philippines, for his unselfish efforts to advance phycological studies in Central Visayas. Professor Reyes (1976:157, pl. 9: figs. 6, 7) previously recorded and described this species as Caulerpa species. His specimens were collected from "dead coral-sandy bottom at the lower intertidal zone" at Siquijor Island, Philippines. 


\section{Literature Cited}

Agardh, C.A.

1817. Synopsis algarum Scandinaviae, adjecta dispositione universali algarum. $\mathrm{xl}+135$ pages. Lund.

1823. Species algarum rite cognitae cum synonymis, differentis specificis et descriptionibus succinctis. Volume 1, part 2, pages 169-521. Lund.

Agardh, J.G.

1837. Novae species algarum, quas in itinere ad oras maris rubri collegit Eduardus Rüppell; cum observationibus. Museum Senckenbergianum, Abhandlungen aus dem Gebiete der Beschreibenden Naturgeschichte, 2:169-174.

1847. Nya alger frän Mexico. Öfversigt af Kongliga Vetenskaps Akademiens Förhandlingar, 4:5-17.

1873. Till algernes systematik, I: Caulerpa., II: Zonaria., III: Sargassum. Lunds Universitets Årsskrift, 9(8): 71 pages.

Blanco, G.J.

1938. Fisheries of Northeastern Luzon and the Babuyan and Batanes Islands. The Philippine Journal of Science, 66(4):501-521, 5 plates.

Børgesen, F.

1907. An Ecological and Systematic Account of the Caulerpas of the Danish West Indies. Kongelige Danske Videnskabernes Selskab Skrifter, Naturvidenske og Mathematik Afd, 4(5):337-392, 31 figures.

Calumpong, H.P.

1981. The Systematics and Distribution of the Benthic Marine Algae of Negros and Adjacent Islands, Philippines. Masters thesis, Silliman University, Dumaguete City 6501, Philippines.

Cordero, P.A., Jr.

1973. On the Marine Algae of Biliran (Leyte) and Vicinities, Central Philippines. Leyte-Samar Studies, Divine Word University, 7(1):15-37.

1974. Distribution of Caulerpa brachypus Harvey (Chlorophyta) in Japan and Adjacent Regions. Nankiseibutu: Nanki Biological Society, 16(1):6-9.

1977. Phycological Observations, V: Gross Morphological Polymorphisms in Caulerpa brachypus (Caulerpales, Chlorophyta) from the Philippines, with Notes on Their Taxonomy. Bulletin of the Japanese Society of Phycology, 25:25-30, 3 figures.

1978. Phycological Observations, VI: Mangrove-associated Algae from Aklan, Philippines. Kalikasan, Philippine Journal of Biology, 7(3):275-296, 5 figures.

Dickie, G.

1877. Contributions to the Botany of the Expedition of
H.M.S. Challenger. Journal of the Linnean Society [of London] (Botany), 15:235-246.

Domantay, J.S.

1962. An Ecological Survey of the Marine Vegetation of the Hundred Islands and Vicinity. The Philippine Journal of Science, 90(2):271-295.

Eubank, L.L.

1946. Hawaiian Representatives of the Genus Caulerpa. University of California Publications in Botany, 18(18):409-432, figures 1, 2, plate 22.

Feldmann, J.

1955. Les plantes des Caulerpa et leur valeur systematique. Revue General de Botanique, 62:422-431, figures $1,2$.

Forsskål, P.

1775. Flora Aegyptiaco-Arabica. 219 pages. Copenhagen.

Galutira, E.C., and G.T. Velasquez

1964. Taxonomy, Distribution and Seasonal Occurrence of Edible Marine Algae in Ilocos Norte, Philippines. The Philippine Journal of Science, 92(4):483522 , figure 1 , plates $1-9$.

Gilbert, W.J.

1942. Notes on Caulerpa from Java and the Philippines. Papers of the Michigan Academy of Sciences, Arts and Letters, 27:7-26, 5 figures.

1946. Studies on Philippine Chlorophyceae, II: Survey of Literature and List of Recorded Species Prior to 1940. Bulletin of the Torrey Botanical Club, 73(1):73-79.

1961. An Annotated Checklist of Philippine Marine Chlorophyta. The Philippine Journal of Science, 88(4):413-449, 1 plate.

Gmelin, S.G.

1768. Historia fucorum. 239 pages, 33 plates. St. Petersburg.

Harvey, W.H.

1860. Characters of New Algae, Chiefly from Japan and Adjacent Regions, Collected by Charles Wright in the North Pacific Exploring Expedition under Captain John Rodgers. Proceedings of the American Academy of Arts and Sciences, 4:327-335.

Howe, M.A.

1905. Phycological Studies, II: New Chlorophyceae, New Rhodophyceae, and Miscellaneous Notes. Bulletin of the Torrey Botanical Club, 32:563-586, plates 23-29.

1932. Marine Algae from the Islands of Panay and Negros (Philippines) and Niuafoou (between Sa- 
moa and Fiji). Journal of the Washington Academy of Science, 22(7):167-170, figure 1.

Kützing, F.T.

1857. Tabulae phycologicae. Volume 7, 40 pages, 100 plates, Nordhausen.

Lamouroux, J.V.

1809a. Mémoires sur les Caulerpes, nouveau genre de la famille des algues marines. Journal de Botanique, 2:136-146.

1809b. Observation sur la physiologie des algues marines, et description de cincq nouveau genres de cette famille. Nouveau Bulletin des Sciences de la Societe Philomatique de Paris, 1:330-333.

Meñez, E.G.

1961. The Marine Algae of the Hundred Islands, Philippines. The Philippine Journal of Science, 90(1):3786,12 plates.

Montagne, J.F.C.

1837. Centurie de plantes cellulaires exotiques nouvelles. Annales des Sciences Naturelles (Botanique), series 2, 8:345-370.

1838. De l'organisation et du mode de reproduction des Caulerpées, et an particulier du Caulerpa Webbiana espèce nouvelle des Iles Canaries. Annales des Sciences Naturelles, Botanique, 9:129-150.

1845. Voyage au Pole Sud et dans l'Oceanie, exécuté par les corvettes l'Astrolabe $e t$ la Zélée. 168 pages, 20 plates. Paris.

Reyes, A.Y.

1972. A Survey of the Littoral Benthic Algae of the Coastal Areas of Dumaguete City. The Philippine Journal of Science, 99(3-4):131-163.

1976. The Littoral Benthic Algae of Siquijor Province, I: Cyanophyta and Chlorophyta. The Philippine Journal of Science, 105(3):133-191, 11 plates.

Taylor, W.R.

1928. The Marine Algae of Florida, with Special Reference to the Dry Tortugas. In Papers from the Tortugas Laboratory, 25. Carnegie Institution of Washington Publication, 379: 219 pages, plates 1-37.

1950. Plants of Bikini and Other Northern Marshall Islands. University of Michigan Studies (Scientific Series), 18: 227 pages, 79 plates.

1966. Records of Asian and Western Pacific Marine Algae, Particularly Algae from Indonesia and the Philippines. Pacific Science, 20(3):342-359, figures $1,2$.

1977. Notes on Plants of the Genus Caulerpa in the Herbarium of Maxwell S. Doty at the University of Hawaii. Atoll Research Bulletin, 208:1-7.

Trono, G.C., Jr.

1972. Annotated Checklist of Some Marine Benthic Al- gae from Tawi-Tawi, Sulu Archipelago. University of the Philippines Natural and Applied Science Bulletin, 24(3):85-112.

1973. The Marine Algae of Siasi Island and Vicinity, I: Introduction and Chlorophyta. Kalikasan, Philippine Journal of Biology, 1:207-228.

1975. The Marine Algae of Bulusan and Vicinity, province of Sorsogon, I: Introduction and Chlorophyta. Kalikasan, Philippine Journal of Biology, 4:2341.

1978. Notes on Some Marine Benthic Algae of Sta. Cruz, Marinduque, Philippines. University of the Philippines Natural Science Research Center Technical Report, 54: 24 pages.

Trono, G.C., Jr., and A. Tuazon

1978. Notes on Some Marine Benthic Algae from Bakawan and Sula Islands, Province of Catanduanes, Philippines. University of the Philippines Natural Science Research Center Technical Report, 53: 19 pages.

Turner, D.

1808. Fuci sive plantarum fucorum generi a botanicis ascriptarum icones, descriptiones et historia. Volume 1, 165 pages, 71 plates. London.

1819. Fuci sive plantarum fucorum generi a botanicis ascriptarum icones, descriptiones et historia. Volume 4, 153 pages, plates 197-258, index 1-7. London.

Vahl, M.

1802. Endeel Kryptogamiske planter (fuci) fra St. Croix. Skrifter af Naturhistorie Selskabet, 5(3):29-47.

Velasquez, G.T.

1953. Seaweed Resources of the Philippines. In Proceedings of the First International Seaweed Symposium, Institute of Seaweed Research, Scotland, pages 100-101.

Velasquez, G.T., D.F. Cornejo, A.E. Santiago, and L. BaensArcega

1973. Algal Communities of Exposed and Protected Marine Waters of Batangas and Bataan. The Philippine Journal of Science, 100(1): 40 pages, 1 figure, 14 plates.

Velasquez, G.T., G.C. Trono, Jr., and M.S. Doty

1975. Algal Species Reported from the Philippines. The Philippine Journal of Science, 101(3-4):115-169.

Weber-van Bosse, A.

1898. Monographie des Caulerpes. Annales du Jardin Botanique de Buitenzorg, 15:243-401, plates 20-34.

Yamada, Y., and T. Tanaka

1938. The Marine Algae from the Island of Yonakuni. Scientific Papers of the Institute of Algological Research, Faculty of Science, Hokkaido Imperial University, 2(1):53-86. 
PLATES 


\section{PLATE 1}

(illustrations reduced to $53 \%$ )

Figure A.-Caulerpa fastigiata, habit, $\times 2$.

Figure B.-Caulerpa cupressoides, habit, $\times 2$.

Figure C.-Same, pinnules, showing 3-ranked branching, $\times 4$.

Figures D., E.-Caulerpa crassifolia, habit, $\times 1$.

FIGURE F.-Caulerpa lentillifera, ramelli, showing imbricate branching and constricted bases, $\times 13$.

Figure G.-Same, habit, $\times 2.6$.

FiguRE H.-Caulerpa arenicola, habit, $\times 3$.

FIGURE I.-Caulerpa brachypus, habit $\times 1.3$. 

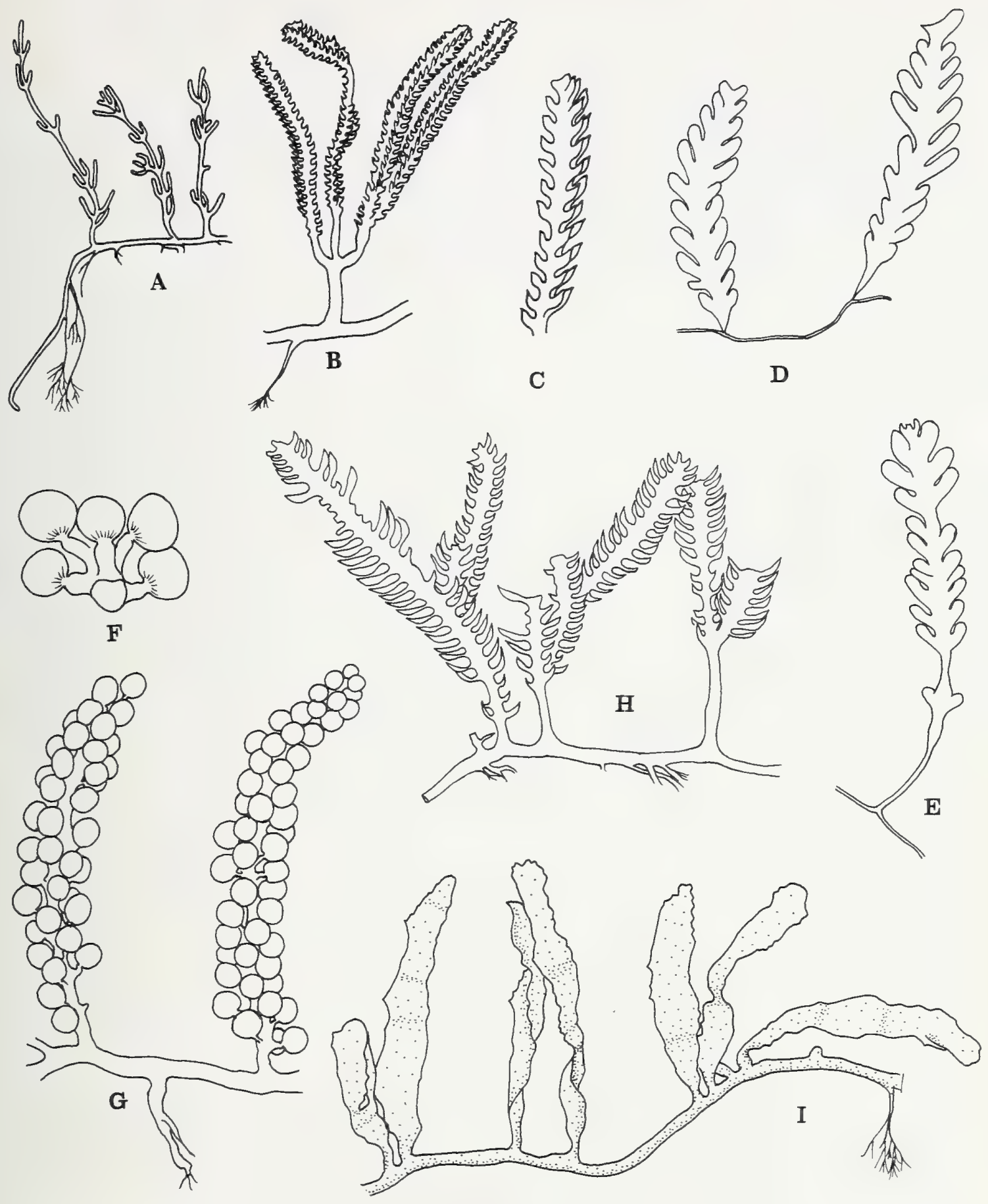


\section{PLATE 2}

(illustrations reduced to $53 \%$ )

Figure A.-Caulerpa racemosa var. clavifera, habit, $\times 2.5$.

Figure B.-Caulerpa racemosa var. occidentalis, habit, $\times 1$.

FigURE C.-Caulerpa racemosa var. macrophysa, habit, $\times 1$.

Figure D.-Caulerpa racemosa var. uvifera, habit, $\times 1$.

Figure E.-Caulerpa serrulata, typical, habit, $\times 1$.

Figure F.-Caulerpa serrulata var. boryana $\mathrm{f}$. occidentalis, habit, $\times 1$.

FIGURE G.-Caulerpa webbiana, habit $\times 2$.

FIGURE H.-Same, erect branch with whorled branchlets, $\times 12$.

FIGURE I.-Same, erect branch with distichous branchlets, $\times 16$.

Figure J.-Same, stolon beset with determinate branchlets, $\times 16$.

FigURE K.-Caulerpa racemosa var. peltata, habit, $\times 2$.

FIGURE L.—Caulerpa microphysa, habit, $\times 2$. 


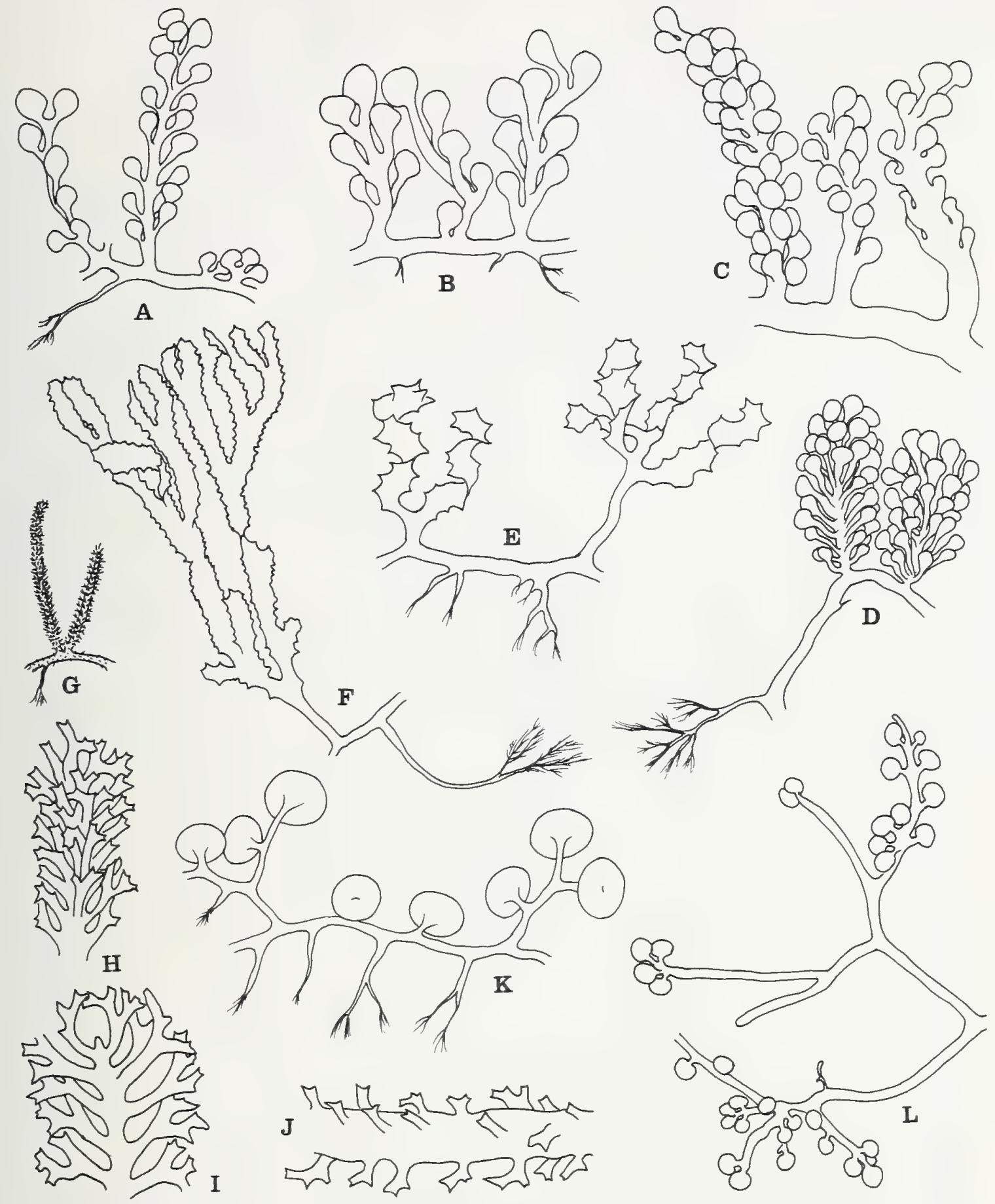




\section{PLATE 3}

(illustrations reduced to $55 \%$ )

Figure A.-Caulerpa verticillata, habit, $\times 3.3$.

Figure B.-Same branchlets with toothed apices, $\times 31$.

FIGURE C.- Same, portion of erect branching showing whorled branchlets, $\times 7$.

FIGURE D.-Caulerpa urvilliana, habit, $\times 1$.

Figure E.-Same, erect branch with mamillate protuberances, $\times 6$.

Figure F.-Caulerpa sertularioides, habit, $\times 2$.

Figure G.-Caulerpa reyesii, new species, habit, $\times 1$.

FigURE H. -Same, erect branch with multi-ranked branchlets, $\times 3.5$.

FIGURE 1.-Same, erect branch with distichous branchlets, $\times 3$. 


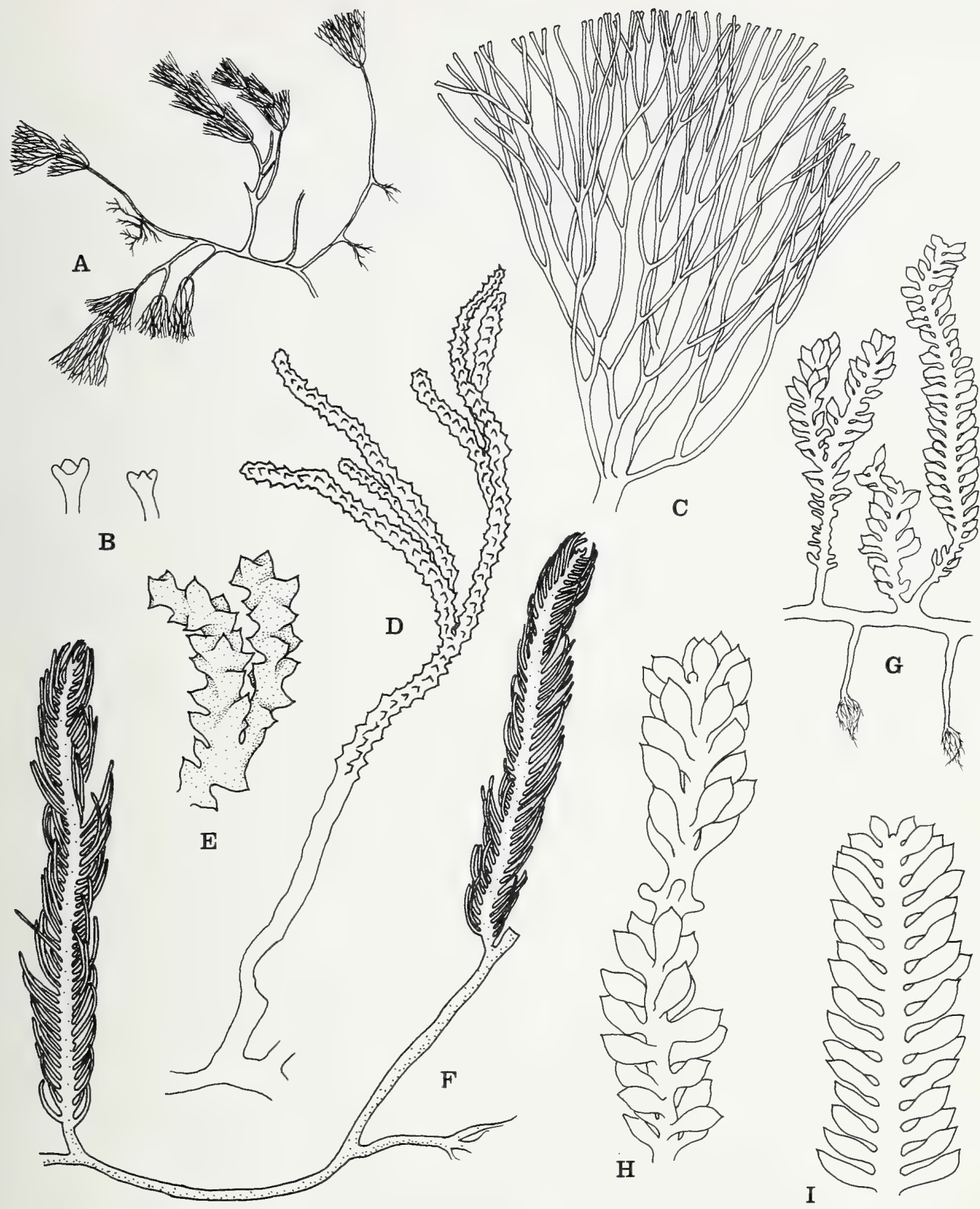







\section{REQUIREMENTS FOR SMITHSONIAN SERIES PUBLICATION}

Manuscripts intended for series publication receive substantive review within their originating Smithsonian museums or offices and are submitted to the Smithsonian Institution Press with approval of the appropriate museum authority on Form SI-36. Requests for special treatment-use of color, foldouts, casebound covers, etc.-require, on the same form, the added approval of designated committees or museum directors.

Review of manuscripts and art by the Press for requirements of series format and style, completeness and clarity of copy, and arrangement of all material, as outlined below, will govern, within the judgment of the Press, acceptance or rejection of the manuscripts and art.

Copy must be typewritten, double-spaced, on one side of standard white bond paper, with 11/4" margins, submitted as ribbon copy (not carbon or xerox), in loose sheets (not stapled or bound), and accompanied by original art. Minimum acceptable length is 30 pages.

Front matter (preceding the text) should include: title page with only title and author and no other information, abstract page with author/title/series/etc., following the established format, table of contents with indents reflecting the heads and structure of the paper.

First page of text should carry the title and author at the top of the page and an unnum bered footnote at the bottom consisting of author's name and professional mailing address.

Center heads of whatever level should be typed with initial caps of major words, with extra space above and below the head, but with no other preparation (such as all caps or underline). Run-in paragraph heads should use period/dashes or colons as necessary.

Tabulations within text (lists of data, often in parallel columns) can be typed on the text page where they occur, but they should not contain rules or formal, numbered table heads.

Formal tables (numbered, with table heads, boxheads, stubs, rules) should be submitted as camera copy, but the author must contact the series section of the Press for editorial attention and preparation assistance before final typing of this matter.

Taxonomic keys in natural history papers should use the alined-couplet form in the zoology and paleobiology series and the multi-level indent form in the botany series. If cross-referencing is required between key and text, do not include page references within the key, but number the keyed-out taxa with their corresponding heads in the text.

Synonymy in the zoology and paleobiology series must use the short form (taxon, author, year:page), with a full reference at the end of the paper under "Literature Cited." For the botany series, the long form (taxon, author, abbreviated journal or book title, volume, page, year, with no reference in the "Literature Cited") is optional.

Footnotes, when few in number, whether annotative or bibliographic, should be typed at the bottom of the text page on which the reference occurs. Extensive notes must appear at the end of the text in a notes section. If bibliographic footnotes are required, use the short form (author/brief title/page) with the full reference in the bibliography.

Text-reference system (author/year/page within the text, with the full reference in a "Literature Cited" at the end of the text) must be used in place of bibliographic footnotes in all scientific series and is strongly recommended in the history and technology series: "(Jones, 1910:122)" or “.. . Jones (1910:122)."

Bibliography, depending upon use, is termed "References," "Selected References," or "Literature Cited." Spell out book, journal, and article titles, using initial caps in all major words. For capitalization of titles in foreign languages, follow the national practice of each language. Underline (for italics) book and journal titles. Use the colon-parentheses system for volume/number/page citations: "10(2):5-9." For alinement and arrangement of elements, follow the format of the series for which the manuscript is intended.

Legends for illustrations must not be attached to the art nor included within the text but must be submitted at the end of the manuscript-with as many legends typed, doublespaced, to a page as convenient.

Illustrations must not be included within the manuscript but must be submitted sepa rately as original art (not copies). All illustrations (photographs, line drawings, maps, etc.) can be intermixed throughout the printed text. They should be termed Figures and should be numbered consecutively. If several "figures" are treated as components of a single larger figure, they should be designated by lowercase italic letters (underlined in copy) on the illustration, in the legend, and in text references: "Figure 9 $\underline{b}$. " If illustrations are intended to be printed separately on coated stock following the text, they should be termed Plates and any components should be lettered as in figures: "Plate $9 \underline{b}$." Keys to any symbols within an illustration should appear on the art and not in the legend.

A few points of style: (1) Do not use periods after such abbreviations as " $m m$, $\mathrm{ft}$, yds, USNM, NNE, AM, BC." (2) Use hyphens in spelled-out fractions: "two-thirds." (3) Spell out numbers "one" through "nine" in expository text, but use numerals in all other cases if possible. (4) Use the metric system of measurement, where possible, instead of the English system. (5) Use the decimal system, where possible, in place of fractions. (6) Use day/month/year sequence for dates: "9 April 1976." (7) For months in tabular listings or data sections, use three-letter abbreviations with no periods: "Jan, Mar, Jun," etc.

Arrange and paginate sequentially EVERY sheet of manuscript-including ALL front matter and ALL legends, etc., at the back of the text-in the following order: (1) title page, (2) abstract, (3) table of contents, (4) foreword and/or preface, (5) text, (6) appendixes, (7) notes, (8) glossary, (9) bibliography, (10) index, (11) legends. 


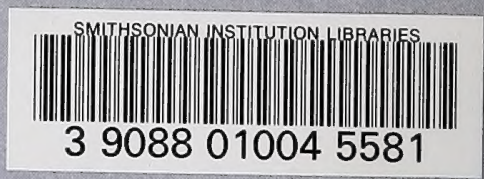

areactostes
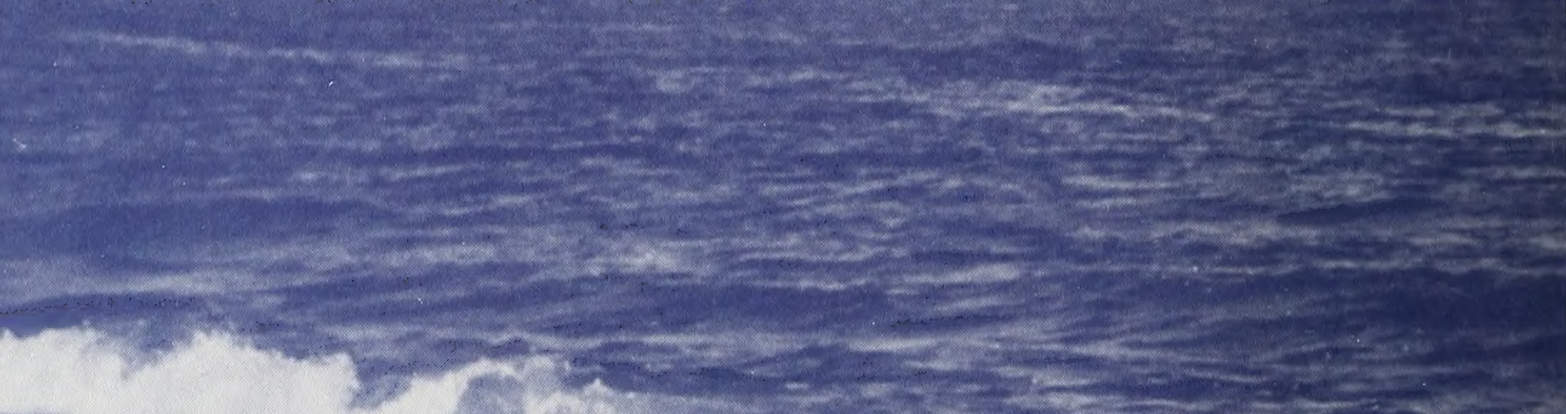

- . 2.
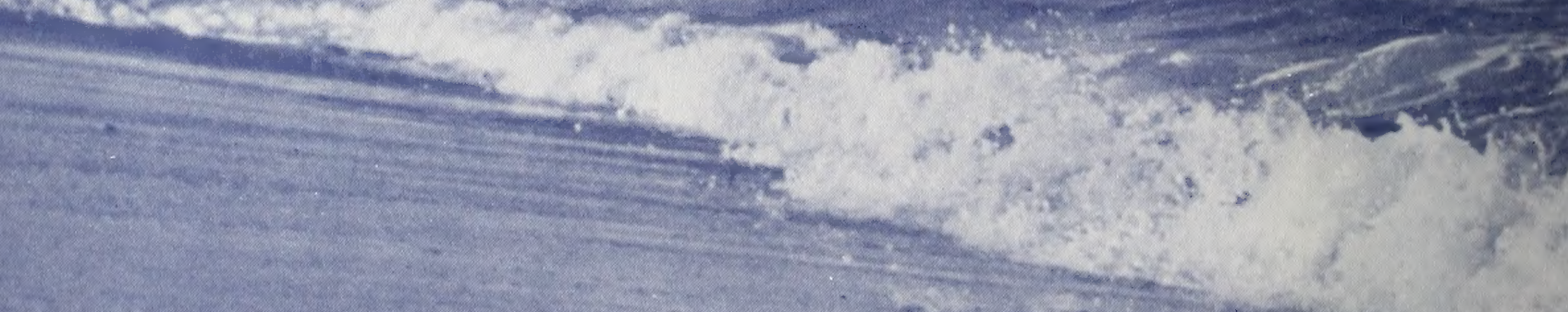